\title{
IceCube neutrino observatory at the South Pole: recent results
}

\author{
Kirill Filimonov for the IceCube Collaboration \\ http://icecube.wisc.edu \\ University of California, Berkeley, CA 94720-7300, USA \\ email: filimonov@berkeley.edu
}

\begin{abstract}
The IceCube neutrino observatory, the largest particle detector in the world $\left(1 \mathrm{~km}^{3}\right)$, is currently being built at the South Pole. IceCube looks down through the Earth to filter out lower-energy particles and uses optical sensors embedded deep in the ultra-clean Antarctic ice to detect high energy neutrinos via Cherenkov radiation from charged particles produced in neutrino interactions. A summary of selected recent results is presented.
\end{abstract}

Keywords. Neutrinos

The upper limit on the flux of high energy neutrinos from optically thin extra-galactic sources can be estimated using the measured flux of high energy $\left(10^{18} \mathrm{eV}\right)$ cosmic rays [Bahcall \& Waxman 2001]. To detect the diffuse flux of neutrinos of $d N / d E_{\nu} \lesssim 5$. $10^{-8} E_{\nu}^{-2} \mathrm{GeV}^{-1} \mathrm{~cm}^{-2} \mathrm{~s}^{-1} \mathrm{sr}^{-1}$ predicted in this way demands very large detector volumes. Both water and ice are naturally occurring optically transparent media for detection of neutrino interactions using Cherenkov radiation. When completed, IceCube will comprise 80 strings of 60 Digital Optical Modules (DOMs) each, deployed between depths of $1,450 \mathrm{~m}$ and 2,450 $\mathrm{m}$ in the clear ice below the Amundsen-Scott Station at the South Pole [Achterberg et al. 2006]. The typical inter-string separation is $125 \mathrm{~m}$ and the DOMs are vertically spaced by $17 \mathrm{~m}$ within each string. The detector geometry is optimized for detection of high-energy neutrinos $\left(E_{\nu}>\mathrm{TeV}\right)$. In addition, 6 closely spaced $(72 \mathrm{~m})$ strings will form a core in the center of the IceCube array, reducing the energy threshold for a subset of the detector volume. On the surface, a pair of ice filled tanks containing two DOMs are placed close to each string to form the IceTop air shower array. The surface array will be used to measure cosmic ray composition and for calibration and background studies. At the time of this conference, IceCube is taking data with 59 strings. Here we concentrate mainly on the data taken with 40 strings during 2008.

\section{Moon Shadow}

As the Earth travels through the interstellar medium, the Moon blocks some cosmic rays from reaching the Earth. This results in a relative deficit of muons measured by IceCube from the direction of the Moon. The resulting deficit (Moon shadow) can be used to calibrate detector angular resolution and pointing accuracy. Figure 1 shows the distribution of reconstructed muons per $1.25^{\circ}$ square bin, relative to the position of the Moon. A $5.2 \sigma$ deficit is observed for the Moon bin. From this, we can conclude that IceCube has no systematic pointing error larger than the search bin, $1.25^{\circ}$ [Boersma et al. 2009]. 




Figure 1. Number of events per $1.25^{\circ}$ square bin, relative to the position of the Moon. The declination of the reconstructed track is within $0.625^{\circ}$ bin from the declination of the Moon.

\section{Point Source Search}

One of the primary physics goals of IceCube is to identify point sources of astrophysical neutrinos. Six months of the IceCube 40-string dataset were unblinded for a point-source search. A maximum likelihood analysis, including an energy term to help separate a hard power-law spectrum from the softer backgrounds, was performed over the whole sky on a very fine grid, along with a list of 39 a priori source candidates. A sky map of directions of 17,777 events (6,796 up-going neutrino candidates and 10,981 high energy down-going muons - the backgrounds for the northern and southern sky searches, respectively) and their significance is presented in Figure 2. The most significant location in the all-sky

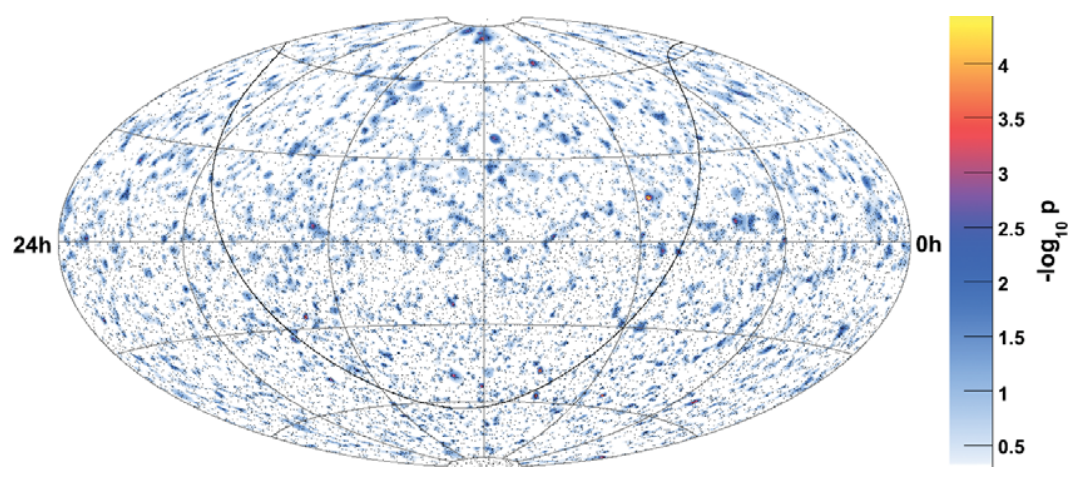

Figure 2. A sky map of the point source candidates (black dots) and their significance (color/shade intensity) in 175.5 days of IC40 data (IceCube preliminary).

search was at r.a. $=114.95^{\circ}$, dec $=15.35^{\circ}$, although an equal or greater significance shows up in 611 out of 1000 scrambled skymaps, meaning the post-trial p-value for the all-sky search is $61.1 \%$. For the list of 39 pre-defined sources, the most significant source was PKS 1622-297 with a pre-trial p-value of 5.2\%. Again from scrambling, the posttrial p-value for the source list was $61.8 \%$. No evidence of a point source of high-energy neutrinos has been found with IceCube so far [Dumm et al. 2009]. Upcoming analyses of a larger dataset with the IC59 and larger configurations will yield much better sensitivity.

\section{References}

Bahcall, J., \& Waxman, E., 2001, Phys. Rev. D 64, 023002

Achterberg, A., et al. IceCube Collaboration, 2006, Astropart. Phys 26, 155

Boersma, D., et al. IceCube Collaboration, 2009, Proc. of the 31st ICRC

Dumm, J., et al. IceCube Collaboration, 2009, Proc. of the 31st ICRC 